\title{
Bacteriological Profile and Clinical Outcome in Cases of Neonatal Sepsis
}

\author{
Renuka Anegundi ${ }^{1}$, Raghavendra D. Kulkarni ${ }^{2}$, Vidyavathi B. Chitharagi ${ }^{3}{ }^{*}$, \\ G.S. Ajantha ${ }^{2}$, C. Shubhada ${ }^{2}$ and Ami Varaiya ${ }^{4}$ \\ ${ }^{1}$ Department of Microbiology, Kasturba Medical College, Manipal, Karnataka, India \\ ${ }^{2}$ Department of Microbiology, SDM College of Medical Sciences and Hospital, Dharwad, \\ Karnataka, India \\ ${ }^{3}$ Department of Microbiology, JSS Medical College, Mysore, Karnataka, India \\ ${ }^{4}$ Infection Control Officer, Nanavati Superspeciality Hospital, Mumbai, Maharashtra, India \\ *Corresponding author
}

\begin{abstract}
A B S T R A C T
Neonatal septicemia is a clinically diagnosed condition having diverse etiology and changing antibiotic sensitivity pattern. The knowledge of common bacterial agents causing septicemia and their antibiogram is useful in deciding empirical antimicrobial therapy. The

Keywords

Blood culture, Gram negative bacilli, Mortality, Neonatal sepsis, Treatment.

Article Info

Accepted: 20 March 2017 Available Online: 10 April 2017 neonatal blood cultures were studied to know the bacterial species and their antibiotic sensitivity pattern. Aim of the study is to evaluate sensitivity pattern of bacterial isolates causing neonatal septicemia and to know clinical outcome of the cases. Automated blood cultures of 250 neonates, clinically diagnosed to have septicemia were performed using BacT/ALERT 3D (BioMerieux). Bottles flagging positive were processed for identification and antibiotic susceptibility tests by Kirby Bauer disc diffusion method. Of the 250 cases studied, blood culture was positive in 117 (46.8\%). Gram positive organisms were predominant (42.2\%), followed by Gram negative organisms (29.7\%). CoNS, Klebsiella pneumoniae, Acinetobacter spp., Escherichia coli were commonest organisms isolated accounting for $29.7 \%, 10.2 \%, 6.2 \%$ and $5.4 \%$ of cases respectively. All gram positive isolates were susceptible to vancomycin and linezolid. Most of gram negative isolates were susceptible to imipenem. Mortality rate was $10.4 \%$ with $65.4 \%$ blood culture positivity. Blood culture helps in rapid and early diagnosis of neonatal sepsis with early shift from empirical therapy to definitive treatment.
\end{abstract}

\section{Introduction}

Children are always precious. Neonatal septicemia is an important and common cause of morbidity and mortality in neonates. World Health Organisation (WHO) estimates about four million neonatal deaths every year worldwide (WHO, 2005). The condition can be rapidly fatal if not treated promptly, aggressively and specifically. Treatment of sepsis is becoming more and more complicated because of increasing antimicrobial resistance. Laboratory evaluation is the only way to reach accurate diagnosis as it is difficult to precisely diagnose the etiology of the condition clinically.

The main objectives of this study includes to identify the sensitivity pattern of the bacterial isolates and to correlate clinical outcome of the cases. 


\section{Materials and Methods}

This study was conducted at tertiary care hospital from October 2010 to July 2011 after taking clearance from institutional ethical committee. Blood samples were collected from 250 clinically diagnosed cases of neonatal septicemia admitted to Neonatal Intensive Care Unit. Sample was directly inoculated into pediatric automated blood collection bottle "pediatric" (yellow, $20 \mathrm{ml}$, BacT/Alert PF). The bottle was loaded in the BacT/ALERT 3D Microbial Detection System (bioMerieux, Durham, North Carolina). After bottle flagged positive, identification of bacteria was done by gram stain, culture and biochemical tests. Sensitivity of the bacterial isolates was performed by Kirby-Bauer's disk diffusion method as per the Clinical Laboratory Standards Institute (CLSI) guidelines (CLSI Guidelines, 2011).ESBL detection was done by disc potentiation test (ceftazidime and ceftazidime - clavulanic acid combination). MRSA were identified by measuring zone around cefoxitin $(30 \mu \mathrm{g})$ disk.

\section{Results and Discussion}

Of the 250 cases studied, 117 cases yielded a positive blood culture giving a yield of $46.8 \%$. A total number of 128 isolates were recovered from 117 positive blood cultures. Bacterial isolates accounted for $71.9 \%$ (92/128) while the yeasts accounted for $28.1 \%(36 / 128)$ isolations.

Among the bacterial isolates, gram positives outnumbered the gram negatives. $42.2 \%$ (54) isolates were gram positive while $29.7 \%$ (38) isolates were gram negative.

MRCoNS was the most common isolate among gram positive organisms (23.4\%), followed by CoNS (6.3\%). Klebsiella pneumoniae was the commonest gram negative isolate accounting for $10.2 \%$, followed by Acinetobacter spp. (6.2\%) and $E$. coli $(5.4 \%)$ (Table 1).

Table 2 shows the sensitivity pattern of Staphylococcal isolates in the study. All the isolates were sensitive to linezolid and vancomycin. Chloramphenicol and clindamycin were the next two better options. From the 30 MRCoNS, 27 were sensitive to chloramphenicol and 25 to clindamycin. Amongst the CoNS, all the eight isolates were sensitive to clindamycin, while seven were resistant to chloramphenicol. Linezolid, vancomycin, clindamycin and chloramphenicol were found to be the best for MRSA.

Table 3 shows in vitro sensitivity pattern of gram negative bacilli isolated from the samples. Imipenem was found to be the most dependable antibiotic. Chloramphenicol was the next antibiotic showing satisfactory sensitivity profile. For most of the other antibiotics, the sensitivity in the organisms was less than $50 \%$.

All the Klebsiella pneumoniae and Enterobacter cloacae strains were found to produce ESBL. For all the other bacilli, 50 or more than $50 \%$ of the strains were ESBL producers (Table 4).

\section{Sensitivity pattern of Streptococcus spp. and Enterococcus spp.}

Both the isolates were only five each in number and were all sensitive to linezolid and vancomycin. Chloramphenicol was effective in $80 \%$ of the isolates ( $4 / 5$ isolates each).

The clinical outcome in all the 250 cases shows that 205 babies recovered and discharged however, 26 of the babies succumbed to infection. The fatality among the cases admitted with septicemia was $10.4 \%$. Of the babies who succumbed to the infection, $65.4 \%$ had positive blood cultures. 
Of the 205 babies recovered, $45.4 \%$ were positive for bacterial and fungal isolates (Table 5).

Sepsis, itself was a cause in nine babies (34.6\%) with $66.6 \%$ blood culture positivity, followed by respiratory distress syndrome (26.9\%) showing $42.8 \%$ positivity. Four babies were categorised under septic shock with $100 \%$ positivity of blood culture and three babies as birth asphyxia.

Of the 26 babies died, 17 had shown positive blood culture. A total of 19 isolates were recovered from 17 blood culture samples. $78.9 \%$ of the isolates were bacterial, of which gram positive bacteria accounted for $36.8 \%$, while gram negative for $42.1 \%$.

Table.1 Bacterial isolates in culture proven sepsis

\begin{tabular}{|c|c|c|c|}
\hline SI. No & $\begin{array}{c}\text { Bacterial isolates } \\
(n=128)\end{array}$ & Number & $\%$ \\
\hline \multicolumn{4}{|c|}{ GRAM POSITIVE COCCI } \\
\hline \multirow[t]{3}{*}{1} & CoNS & 38 & 29.6 \\
\hline & MRCoNS & (30) & $(23.4)$ \\
\hline & CoNS & (8) & (6.3) \\
\hline 2 & MRSA & 6 & 4.7 \\
\hline 3 & Streptococcus spp. & 5 & 3.9 \\
\hline \multirow[t]{2}{*}{4} & Enterococcus spp. & 5 & 3.9 \\
\hline & Total & 54 & 42.2 \\
\hline \multicolumn{4}{|c|}{ GRAM NEGATIVE BACILLI } \\
\hline 1 & Klebsiella pneumoniae & 13 & 10.2 \\
\hline 2 & Acinetobacterspp. & 8 & 6.2 \\
\hline 3 & E. coli & 7 & 5.4 \\
\hline 4 & Pseudomonas aeruginosa & 4 & 3.2 \\
\hline 5 & Enterobacter cloacae & 3 & 2.4 \\
\hline 6 & NFGNB & 2 & 1.6 \\
\hline \multirow[t]{3}{*}{7} & Sphingomonas spp. & 1 & 0.8 \\
\hline & Total & 38 & 29.7 \\
\hline & Yeasts & 36 & 28.1 \\
\hline
\end{tabular}

MRCoNS was the most common isolate among gram positive organisms (23.4\%), followed by CoNS (6.3\%). Klebsiella pneumoniae was the commonest gram negative isolate accounting for $10.2 \%$, followed by Acinetobacter spp. $(6.2 \%)$ and E. coli $(5.4 \%)$. 
Int.J.Curr.Microbiol.App.Sci (2017) 6(4): 2206-2214

Table.2 Sensitivity of Staphylococcal isolates

\begin{tabular}{|l|c|c|c|}
\hline \multicolumn{5}{|c|}{ Sensitivity of Staphylococcal isolates } \\
\hline & $\begin{array}{c}\text { MRCoNS (\%) } \\
(\boldsymbol{n}=\mathbf{3 0})\end{array}$ & $\begin{array}{c}\text { CoNS (\%) } \\
(\boldsymbol{n = 8})\end{array}$ & $\begin{array}{c}\text { MRSA (\%) } \\
(\boldsymbol{n = 6})\end{array}$ \\
\hline Penicillin & $0(0 \%)$ & $2(25 \%)$ & $0(0 \%)$ \\
\hline Erythromycin & $5(16.7 \%)$ & $5(62.5 \%)$ & $2(33.3 \%)$ \\
\hline Clindamycin & $25(83.3 \%)$ & $8(100 \%)$ & $6(100 \%)$ \\
\hline Gentamicin & $5(16.7 \%)$ & $4(50 \%)$ & $1(16.7 \%)$ \\
\hline Tetracycline & $16(53.3 \%)$ & $2(25 \%)$ & $5(83.3 \%)$ \\
\hline Ciprofloxacin & $18(60 \%)$ & $6(75 \%)$ & $2(33.3 \%)$ \\
\hline Cotrimoxazole & $11(36.7 \%)$ & $4(50 \%)$ & $2(33.3 \%)$ \\
\hline Chloramphenicol & $27(90 \%)$ & $7(87.5 \%)$ & $6(100 \%)$ \\
\hline Vancomycin & $30(100 \%)$ & $8(100 \%)$ & $6(100 \%)$ \\
\hline Linezolid & $30(100 \%)$ & $8(100 \%)$ & $6(100 \%)$ \\
\hline
\end{tabular}

Table.3 Sensitivity pattern among gram negative bacilli

\begin{tabular}{|l|c|c|c|c|c|c|c|}
\hline & Kp $(\boldsymbol{n}=\mathbf{1 3})$ & Aci $(\boldsymbol{n}=\mathbf{8})$ & $\mathbf{E c}(\boldsymbol{n}=7)$ & Ps $(\boldsymbol{n}=\mathbf{4})$ & Ecl $(\boldsymbol{n}=\mathbf{3})$ & $\begin{array}{c}\text { NFGNB } \\
(\boldsymbol{n}=2)\end{array}$ & $\mathbf{S}(\boldsymbol{n}=\mathbf{1})$ \\
\hline IPM & $11(84.6 \%)$ & $5(62.5 \%)$ & $7(100 \%)$ & $3(75 \%)$ & $3(100 \%)$ & $1(50 \%)$ & $1(100 \%)$ \\
\hline AT & $0(0 \%)$ & - & $3(42.9 \%)$ & $1(25 \%)$ & $0(0 \%)$ & $1(50 \%)$ & $1(100 \%)$ \\
\hline CTX & $0(0 \%)$ & $0(0 \%)$ & $2(28.6 \%)$ & - & $0(0 \%)$ & $1(50 \%)$ & $1(100 \%)$ \\
\hline CX & $0(0 \%)$ & - & $2(28.6 \%)$ & - & $0(0 \%)$ & $1(50 \%)$ & $1(100 \%)$ \\
\hline CTR & $0(0 \%)$ & $0(0 \%)$ & $3(42.9 \%)$ & - & $0(0 \%)$ & $1(50 \%)$ & $1(100 \%)$ \\
\hline CAZ & $0(0 \%)$ & $0(0 \%)$ & $3(42.9 \%)$ & $1(25 \%)$ & $0(0 \%)$ & $1(50 \%)$ & $1(100 \%)$ \\
\hline CAC & $2(15.4 \%)$ & $1(12.5 \%)$ & $4(57.1 \%)$ & - & $1(33.3 \%)$ & $1(50 \%)$ & $1(100 \%)$ \\
\hline AMP & $0(0 \%)$ & - & $1(14.3 \%)$ & - & $2(66.7 \%)$ & $0(0 \%)$ & $1(100 \%)$ \\
\hline CXM & $0(0 \%)$ & - & $2(28.6 \%)$ & - & $0(0 \%)$ & $1(50 \%)$ & $1(100 \%)$ \\
\hline CIP & $6(46.2 \%)$ & $3(37.5 \%)$ & $5(71.4 \%)$ & $2(50 \%)$ & $2(66.7 \%)$ & $2(100 \%)$ & $1(100 \%)$ \\
\hline GEN & $2(15.4 \%)$ & $0(0 \%)$ & $2(28.6 \%)$ & $1(25 \%)$ & $0(0 \%)$ & $1(50 \%)$ & $0(0 \%)$ \\
\hline AK & $3(23.1 \%)$ & $0(0 \%)$ & $3(42.9 \%)$ & $2(50 \%)$ & $0(0 \%)$ & $1(50 \%)$ & $1(100 \%)$ \\
\hline TE & $1(7.7 \%)$ & $2(25 \%)$ & $3(42.9 \%)$ & - & $0(0 \%)$ & $2(100 \%)$ & $1(100 \%)$ \\
\hline COT & $5(38.5 \%)$ & $2(25 \%)$ & $2(28.6 \%)$ & - & $3(100 \%)$ & $1(50 \%)$ & $1(100 \%)$ \\
\hline PI & $0(0 \%)$ & $1(12.5 \%)$ & $2(28.6 \%)$ & $1(25 \%)$ & $0(0 \%)$ & $1(50 \%)$ & $1(100 \%)$ \\
\hline CPM & $0(0 \%)$ & $0(0 \%)$ & $3(42.9 \%)$ & $1(25 \%)$ & $0(0 \%)$ & $1(50 \%)$ & $1(100 \%)$ \\
\hline C & $9(69.2 \%)$ & - & $5(71.4 \%)$ & - & $2(66.7 \%)$ & $1(50 \%)$ & $1(100 \%)$ \\
\hline
\end{tabular}


Table.4 ESBL production

\begin{tabular}{|l|c|c|c|}
\hline \multicolumn{4}{|c|}{ ESBL production } \\
\hline \multicolumn{1}{|c|}{ Organism } & Number & ESBL & $\%$ \\
\hline Klebsiella pneumoniae & 13 & 13 & 100.0 \\
\hline E. coli & 7 & 4 & 57.1 \\
\hline Enterobacter cloacae & 3 & 3 & 100.0 \\
\hline Pseudomonas aeruginosa & 4 & 2 & 50.0 \\
\hline NFGNB & 2 & 1 & 50.0 \\
\hline Sphingomonas spp. & 1 & 0 & 0.0 \\
\hline
\end{tabular}

Acinetobacter spp. was not tested for ESBL production

Table.5 Clinical outcome of the cases

\begin{tabular}{|l|c|c|c|c|c|c|}
\hline $\begin{array}{c}\text { Clinical } \\
\text { outcome }\end{array}$ & Number & $\boldsymbol{\%}$ & $\begin{array}{c}\text { Culture } \\
\text { Positive }\end{array}$ & $\boldsymbol{\%}$ & $\begin{array}{c}\text { Culture } \\
\text { negative }\end{array}$ & $\%$ \\
\hline Death & 26 & 10.4 & 17 & 65.4 & 9 & 34.6 \\
\hline Recovered & 205 & 82.0 & 93 & 45.4 & 112 & 54.6 \\
\hline DAMA & 19 & 7.6 & 7 & 36.8 & 12 & 63.2 \\
\hline Total & $\mathbf{2 5 0}$ & $\mathbf{1 0 0}$ & $\mathbf{1 1 7}$ & - & $\mathbf{1 3 3}$ & - \\
\hline
\end{tabular}

Table.6 Cause of death

\begin{tabular}{|c|c|c|c|}
\hline Cause of death & Number & \% & Blood culture positive (\%) \\
\hline Sepsis & 9 & 34.6 & $66.6 \%$ \\
\hline Respiratory distress syndrome & 7 & 26.9 & $42.8 \%$ \\
\hline Septic Shock & 4 & 15.4 & $100 \%$ \\
\hline Birth asphyxia & 3 & 11.5 & $33.3 \%$ \\
\hline $\begin{array}{c}\text { Others (occipital encephalitis, } \\
\text { congenital heart disease and } \\
\text { congenital anomaly) }\end{array}$ & 3 & 11.5 & $33.3 \%$ \\
\hline Total & $\mathbf{2 6}$ & $\mathbf{1 0 0}$ & \\
\hline \multicolumn{2}{|c|}{} \\
\hline
\end{tabular}


Table.7 Organisms responsible for death

\begin{tabular}{|c|c|c|}
\hline \multicolumn{1}{|c|}{ Organism } & Death & $\mathbf{\%}$ \\
\hline Bacteria & $\mathbf{1 5}$ & $\mathbf{7 8 . 9}$ \\
\hline Gram positive & $\mathbf{7}$ & 36.8 \\
\hline MRSA & 2 & 10.5 \\
\hline MRCoNS & 2 & 10.5 \\
\hline Streptococcus spp. & 2 & 10.5 \\
\hline Enterococcus spp. & 1 & 5.3 \\
\hline Gram-negative & $\mathbf{8}$ & $\mathbf{4 2 . 1}$ \\
\hline E. coli & 3 & 15.8 \\
\hline Acinetobacter spp. & 2 & 10.5 \\
\hline Klebsiella pneumoniae & 1 & 5.3 \\
\hline E. cloacae & 1 & 5.3 \\
\hline Pseudomonas aeruginosa & 1 & 5.3 \\
\hline
\end{tabular}

Neonatal sepsis is a life threatening emergency and any delay in treatment may lead to fatality. The organisms causing neonatal sepsis vary from hospital to hospital and from one community to other. These differences could be due to the characteristics of the population, their religious faiths, customs and habits, pattern of antimicrobial agents used and local and nursery practices. Sepsis contributes to $30-50 \%$ of neonatal deaths in developing countries. Ballot et al., (2012) showed CoNS to be the predominant organisms accounting for $19.1 \%$ followed by Klebsiella pneumoniae $12.1 \%$ and Acinetobacter spp. 10.9\%. Ghotaslou et al., (2007), showed CoNS as the most frequent isolate followed by Klebsiella pneumoniae $(16.6 \%)$.

The study done by Macharashvili et al., (2009), showed $100 \%$ sensitivity to vancomycin for $S$. aureus and $S$. epidermidis isolates. Ballot et al., (2012), reported a similar picture with $100 \%$ vancomycin sensitivity. The emergence of MRSA poses a serious threat to use of routinely administered antimicrobials which necessitates the use of vancomycin as the useful drug for management of these cases. Vancomycin is an exclusive drug in the treatment of gram positive infections especially against MRSA and Enterococci. In the present study, 
linezolid has shown excellent efficacy that is complete absence of resistance in staphylococci including MRSA.

Vancomycin needs to be protected. It should be used exceptionally. Linezolid which is well tolerated in neonates is a better alternative. Therefore, for multi drug resistant staphylococcal infections linezolid may be considered instead of vancomycin. Coagulase negative Staphylococci are emerging as causative organisms of neonatal septicemia and recovery of these pathogens should no longer be considered as a contaminant especially in preterm and low birth weight babies with longer hospital stay and exposure to diagnostic and supportive procedures (Baptiste et al., (2011).

The present study showed the emerging carbapenem resistance in the gram negative bacilli. The study by Viswanathan et al., (2008) also showed $6.6 \%$ of emerging carbapenem resistance among gram negative isolates. In that study, Gram-negative organisms showed $100 \%$ resistance to ampicillin, cefotaxime, and gentamicin. According to Vinodkumar et al., (2008), majority of gram negative organisms showed resistance to one or more antibiotics. According to the study done by Prabhu et al., (2003), gram negative bacilli showed high resistance to ampicillin (64.28\%). Cefotaxime or ceftazidime with amikacin was the most appropriate drug for multi resistant strains of gram negative bacilli.

Resistant bacteria are emerging worldwide as a real threat to the favourable outcome of common infections in community and hospital settings. High morbidity, prolonged hospital stay as well as high mortality are observed in resistant gram negative infections. There is a tendency among clinicians to directly begin the therapy with carbapenems like imipenem. A variety of mechanisms like ESBL production, Amp - C, metallo $\beta$-lactamase etc, have led us to a frightening situation. Organisms harbouring multiple mechanisms simultaneously are not uncommon. Acquisition of carbapenem resistance by recalcitrant organisms has created pan-resistant strains. Pan resistance is not a mere fiction but is a reality being experienced by many tertiary care centres. In the present study, we encountered two Klebsiella pneumoniae, three Acinetobacter spp. and one Pseudomonas aeruginosa and one NFGNB that were resistant to imipenem. This is a clear indication of futility of using carbapenems for empirical therapy for septicemia.

According to Shakil et al., (2010), 36.5\% of Klebsiella pneumoniae and $28.6 \%$ of E. coli isolates were ESBL producers. ${ }^{10}$ Sehgal et al., (2007) showed that $61.3 \%$ of gram negative bacteria were showing ESBL production. The problem of antibiotic resistance is worsening worldwide. The widespread and injudicious use of broad-spectrum beta-lactam antibiotics has led to a marked increase in the incidence of ESBL-producing gram-negative organisms. This has necessitated the use of expensive drugs such as carbapenems. Thus, management of such cases is a major challenge to the clinicians.

According to Viswanathan et al., (2008), out of 216 suspected cases, 29 babies died with mortality rate of $13.4 \%{ }^{7}$ According to Chacko et al., (2005), case fatality rate was $19.4 \%$ with $13.3 \%$ culture positivity rate. Early positive blood culture helps us to identify high risk babies.

According to Khatun et al., (2012), birth asphyxia was the main cause contributing to $42 \%$ followed by sepsis and pneumonia (27\%) and birth trauma (7\%). The study done by Lahariya et al., (2010), showed $24.9 \%$ of deaths due to sepsis and pneumonia followed 
by asphyxia in $18.5 \%$ and prematurity/LBW in $16.8 \%$ of cases. Neonatal sepsis is the single most important cause of death in the community. Sepsis related neonatal deaths are preventable if diagnosed and treated early.

According to the study done by Sriram et al., (2011), 57.1\% of deaths were caused by gram negative organisms in late onset sepsis cases and $44.4 \%$ of the deaths were caused by gram positive organisms in early onset sepsis. According to the study done by Ballot et al., (2012), mortality was significantly associated with gram negative organisms. Study by Jun Ho Wu et al., (2009), showed that high mortality rate was contributed by Candida spp. (15\%) followed by gram positive and gram negative organisms $(8 \%$ and $7 \%)$ respectively. The majority of the death was contributed by gram negative bacilli, which were multi resistant to commonly used drugs, indicating their high degree of virulence (Tables 6 and 7).

Conclusions of the study are as follows

Antimicrobial agents are the most important tool for managing infectious diseases. The wide availability of antibiotics and inappropriate use of broad spectrum antibiotics in the community has led to the development of multidrug resistant bacteria. The antibiotic resistance is a global problem. The emerging multidrug resistant organisms like Acinetobacter spp., ESBL, AmpC, metallo $\beta$-lactamase producing gram negative bacteria pose a threat in treatment of infections due to these isolates.

The number of Acinetobacter spp. recorded in the present study were eight, accounting for $6.2 \%$ of the isolates. Two babies succumbed to the infection. Though the number is small, $25 \%$ of the Acinetobacter spp. infected babies could not be saved. High vigilance to rapidly identify these infections is critical in the management of neonatal sepsis.

There is requirement of simple and sustainable intervention to reduce the burden of multidrug resistant pathogens causing sepsis. The implementation of hand washing, barrier nursing and antibiotic stewardship policies is need of time.

\section{References}

Ballot, D.E., T. Nana, C. Sriruttan, and P.A. Cooper. 2012. Bacterial Bloodstream Infections in Neonates in a Developing Country. Int. Scholarly Res. Network Pediatrics, 1-6.

Baptiste, N.J., D.K. Benjamin, Jr., M. CohenWolkowiez, V.G. Fowler Jr., M. Laughon, R.H. Clark, and P. Brian Smith. 2011. Coagulase-negative Staphylococcal Infections in the Neonatal Intensive Care Unit. Infect. Control Hosp. Epidemiol., 32(7): 679686.

Chacko, B., and I. Sohi. 2005. Early Onset Neonatal Sepsis. Indian J. Paediatrics, 72: 23-26.

CLSI. 2011. Performance Standards for Antimicrobial Disk Susceptibility Tests; Twenty first information Supplement. CLSI document M100-S22. Pennsylvania, USA: Clinical and Laboratory Standards Institute.

Ghotaslou, R., Z. Ghorashi, and M. Nahaei. 2007. Klebsiella pneumoniae in Neonatal Sepsis: A 3-Year-Study in the Pediatric Hospital of Tabriz, Iran. Jpn. J. Infect. Dis., 60: 126-128.

Jun-Ho Wu, Chien-Yi Chen, Po-Nien Tsao, Wu-Shiun Hsieh, and Hung-Chieh Chou. 2009. Neonatal Sepsis: A 6-Year Analysis in a Neonatal Care Unit in Taiwan. Pediatr. Neonatol., 50(3): 88-95.

Khatun, F., Rasheed, S., Moran, A.C., Alam, 
A.M., Shomik, M.S., Sultana, M., Choudhury, N., Iqbal, M., and Bhuiya, A. 2012. Causes of neonatal and maternal deaths in Dhaka slums: Implications for service delivery. $B M C$ Public Health, 12: 84.

Lahariya, C., C.R. Sudfeld, D. Lahariya, and S.S. Tomar. 2010. Causes of child deaths in India, 1985-2008: a systematic review of literature. Indian J. Pediatr., 77(11): 1303-1311.

Macharashvili, N., E. Kourbatova, M. Butsashvili, T. Tsertsvadze, L. McNutt, and M. Leonard. 2009. Etiology of neonatal blood stream infections in Tbilisi, Republic of Georgia. Int. J. Infect. Dis., 13: 499-505.

Prabhu, K., S. Bhat and S. Rao. 2010. Bacteriologic profile and antibiogram of blood culture isolates in a pediatric care unit. J. Lab. Physicians, 2(2): 85-88.

Shakil, S., S.Z. Ali, M. Akram, S.M. Ali, and A.U. Khan. 2010. Risk Factors for Extended-Spectrum $\quad \beta$-Lactamase Producing Escherichia Coli and Klebsiella Pneumoniae Acquisition in a Neonatal Intensive Care Unit. J. Trop. Pediatrics, 56(2): 90-96.
Sehgal, R., R. Gaind, H. Chellani, and P. Agarwal. 2007. Extended-spectrum beta lactamase-producing gram-negative bacteria: clinical profile and outcome in a neonatal intensive care unit. Ann. Trop. Paediatr., 27(1): 45-54.

Viswanathan, R., A.K. Singh, C. Ghosh, S. Dasgupta, S. Mukherjee, S. Basu. 2012. Profile of Neonatal Septicaemia at a District-level Sick Newborn Care Unit. J. Health Popul. Nutr., 30(1): 41-48.

Vinodkumar, C.S., Y.F. Neelagund, S. Kalsurmath, S. Banapurmath, N.K. Kalappannavar and K.G. Basavarajappa. 2008. Perinatal risk factors and microbial profile of neonatal septicemia: A multicentred study. Obstet. Gynecol. India, 58(1): 32-40.

Sriram, R. 2011. Correlation of Blood culture results with the Sepsis score and the Sepsis screen in the diagnosis of Neonatal Septicemia. Int. J. Biol. Med. Res., 2(1): 360-368.

World Health Organization. 2005. Make Every Mother and Child Count. Chapter 5: Newborns: no longer going unnoticed. World Health Report: Geneva.

\section{How to cite this article:}

Renuka Anegundi, Raghavendra D. Kulkarni, Vidyavathi B. Chitharagi, G.S. Ajantha, C. Shubhada and Ami Varaiya. 2017. Bacteriological Profile and Clinical Outcome in Cases of Neonatal Sepsis. Int.J.Curr.Microbiol.App.Sci. 6(4): 2206-2214. doi: https://doi.org/10.20546/ijcmas.2017.604.258 